\title{
GROUPS OF MOTIONS IN CONFORMALLY FLAT SPACES. II
}

\section{JACK LEVINE}

1. Introduction. In a previous paper with a similar title,* we have shown that all groups of motions admitted by a conformally flat metric space $V_{n}$ must be subgroups of the general conformal group $G_{N}$ of $N=\frac{1}{2}(n+1)(n+2)$ parameters generated by $\dagger$

$$
\xi^{i}=b^{i}+a_{0} x^{i}+x^{i} a_{j} x^{j}-\frac{1}{2} a_{i} e_{i} e_{j}\left(x^{j}\right)^{2}+b_{j}^{i} x^{j}, \quad e_{i}= \pm 1 .
$$

In (1), the $b_{j}{ }^{i}$ satisfy the relations $e_{i} b_{j}{ }^{i}+e_{j} b_{i}{ }^{j}=0,(i, j$ not summed $)$. Otherwise the $a$ 's and $b$ 's in (1) are arbitrary.

To define a group of motions of $V_{n}$, the $\xi^{i}$ must satisfy the equations

$$
\xi^{k} \frac{\partial h}{\partial x^{k}}+h \frac{\partial \xi^{i}}{\partial x^{i}}=0, \quad i \text { not summed, }
$$

and the coordinates $x^{i}$ of (2) are such that $g_{i j}=e_{i} \delta_{j}{ }^{i} h^{2}$. Hence in this coordinate system, the metric has the form

$$
d s^{2}=h^{2} \sum e_{i}\left(d x^{i}\right)^{2} .
$$

In this paper we shall consider the simplest subgroups of $G_{N}$, and determine the nature of the function $h$ corresponding to each. Also we give a restatement of Theorem 2 of I, since it is not complete as given.

2. The group $G_{N}$. The basis of the group $G_{N}$ may be taken in the form

$$
\begin{aligned}
P_{i} & =p_{i}, \\
S_{i j} & =e_{i} x^{i} p_{j}-e_{j} x^{i} p_{i}, \quad i, j \text { not summed, } \\
U & =x^{i} p_{i}, \\
V_{i} & =2 x^{i} x^{i} p_{j}-e_{i} e_{j}\left(x^{j}\right)^{2} p_{i},
\end{aligned}
$$

where $p_{i}=\partial / \partial x^{i}$; and its commutators are $\ddagger$

* Groups of motions in conformally fat spaces, this Bulletin, vol. 42 (1936), pp. 418-422. The results of this paper (which we refer to as I) will be assumed known.

$\dagger$ All small Latin indices take the values $1,2, \cdots, n$, with $n>2$, unless otherwise noted.

¥ S. Lie, Theorie der Transformationsgruppen, vol. 3, pp. 321-334. 


$$
\begin{aligned}
\left(P_{i}, P_{j}\right) & =0 \\
\left(P_{i}, U\right) & =P_{i} \\
\left(P_{i}, S_{j k}\right) & =e_{i} \delta_{i j} P_{k}-e_{k} \delta_{i k} P_{j}, \\
\left(P_{i}, V_{j}\right) & =2 \delta_{i j} U-2 e_{j} S_{i j} \\
\left(S_{i j}, S_{k l}\right) & =e_{j} \delta_{j k} S_{i l}-e_{j} \delta_{j} S_{i k}-e_{i} \delta_{i k} S_{j l}+e_{i} \delta_{i} S_{j k}, \\
\left(S_{i j}, U\right) & =0 \\
\left(S_{i j}, V_{k}\right) & =e_{i} \delta_{j k} V_{i}-e_{j} \delta_{i k} V_{j}, \\
\left(U, V_{i}\right) & =V_{i} \\
\left(V_{i}, V_{j}\right) & =0 .
\end{aligned}
$$

The four types of symbols, $P_{i}, S_{i j}, U, V_{i}$, will be considered singly and in various combinations to form the subgroups to be discussed.

3. Subgroups of one type of symbol. We consider first the subgroups with symbols*
(a) $\left[P_{\alpha}\right]$,
(b) $[U]$,
(c) $\left[S_{\alpha \beta}\right]$,
(d) $\left[V_{\alpha}\right]$.

The notation $\left[P_{\alpha}\right]$ means $\left[P_{1}, P_{2}, \cdots, P_{r}\right]$, and similarly for other expressions of this nature. That each of (a)-(d) forms a subgroup follows from (8a), (8e), (8i).

For (a), we have from (4), $\xi_{\alpha}^{i}=\delta_{\alpha}^{i}$, and (2), written in the form

becomes

$$
\xi_{\alpha}^{k} \frac{\partial h}{\partial x^{k}}+h \frac{\partial \xi_{\alpha}^{i}}{\partial x^{i}}=0, \quad i \text { not summed, }
$$

$$
\frac{\partial h}{\partial x^{\alpha}}=0 .
$$

Hence $(\mathrm{a}): h=h\left(x^{r+1}, \cdots, x^{n}\right)$. In case $r=n, h$ is constant, and the $V_{n}$ is flat.

The finite equations of the group $\left[P_{\alpha}\right]$ are

$$
x^{i}=x^{i}+a^{\alpha} \delta_{\alpha}^{i}
$$

with parameters $a^{\alpha}$. Because of the form of (10), we call this group the $T_{r}$ of translations. However, the group of motions $\left[P_{\alpha}\right]$ is not a group of translations of the $V_{n}$ unless $h=$ constant, $\dagger$ that is, unless $V_{n}$ is flat.

* Greek letters take the values $1,2, \cdots, r$, with $r \leqq n$.

$\dagger$ L. P. Eisenhart, Continuous Groups of Transformations, p. 212. We refer to this book as CG. 
For (b), we have $\xi^{i}=x^{i}$, and (2) becomes

$$
x^{i} \frac{\partial h}{\partial x^{i}}=-h .
$$

Hence $h$ is homogeneous of degree -1 , that is,

$$
h=\frac{1}{x^{1}} \phi\left(\frac{x^{2}}{x^{1}}, \ldots, \frac{x^{n}}{x^{1}}\right),
$$

say, where $\phi$ is an arbitrary function of its arguments.

The finite equations of the group $[U]$ are $x^{\prime i}=a x^{i}$, the group of dilations.

For (c), we find

$$
\stackrel{i}{\xi}_{\alpha \beta}=e_{\alpha} \delta_{\beta}^{i} x^{\alpha}-e_{\beta} \delta_{\alpha}^{i} x^{\beta},
$$$$
\alpha \neq \beta,
$$

as the vector components of the group $\left[S_{\alpha \beta}\right]$ of $\frac{1}{2} r(r-1)$ parameters. The equations (2) which must be satisfied for each $\xi_{\alpha \beta}^{i}$ now become

$$
X_{\alpha \beta} h \equiv e_{\alpha} x^{\alpha} \frac{\partial h}{\partial x^{\beta}}-e_{\beta} x^{\beta} \frac{\partial h}{\partial x^{\alpha}}=0, \quad \alpha, \beta \text { not summed }
$$

These equations have as general solution,

$$
h=h\left(u ; x^{r+1}, \cdots, x^{n}\right),
$$

where $u=\sum e_{\alpha}\left(x^{\alpha}\right)^{2}$.

In obtaining this, we use the fact that the system (12) contains $r-1$ independent equations, since

$$
e_{\alpha} x^{\alpha} X_{\beta \gamma}+e_{\beta} x^{\beta} X_{\gamma \alpha}+e_{\gamma} x^{\gamma} X_{\alpha \beta} \equiv 0, \quad \text { no summing, }
$$

and it is also a complete system.*

The group $\left[S_{\alpha \beta}\right]$ has the finite equations

$$
x^{\prime \alpha}=a_{\beta}{ }^{\alpha} x^{\beta}, \quad x^{A}=x^{A}, \quad A=r+1, \cdots, n,
$$

with

$$
\sum e_{\alpha} a_{\beta}^{\alpha} a_{\gamma}^{\alpha}=e_{\beta} \delta_{\beta \gamma} .
$$

We call this group of $\frac{1}{2} r(r-1)$ parameters, the $R_{r(r-1) / 2}$ of rotations. $\dagger$ The vector components for the group (d) are

$$
\xi_{\alpha}^{i}=2 x^{i} x^{\alpha}-e_{\alpha} \delta_{\alpha}^{i} R,
$$

* Goursat, Mathematical Analysis, vol. 2, part 2, p. 270.

† CG, p. 57, problem 12 . 
where $R=\sum e_{i}\left(x^{i}\right)^{2}$. Equations (2) reduce, for this case, to

$$
2 x^{\alpha} x^{i} \frac{\partial h}{\partial x^{i}}-e_{\alpha} R \frac{\partial h}{\partial x^{\alpha}}+2 h x^{\alpha}=0 .
$$

If we put $\dot{\lambda}=x^{i} \partial h / \partial x^{i}$, (13) may be written in the form

$$
\frac{2(\lambda+h)}{R}=\frac{e_{\alpha}}{x^{\alpha}} \frac{\partial h}{\partial x^{\alpha}},
$$

$\alpha$ not summed.

Since the left member of this equation is independent of $\alpha$, we may write

$$
\frac{e_{\alpha}}{x^{\alpha}} \frac{\partial h}{\partial x^{\alpha}}=\frac{e_{\beta}}{x^{\beta}} \frac{\partial h}{\partial x^{\beta}}
$$

which simplifies to (12), and hence $h$ is of the form for (c). Using this form for $h$ in (13), we obtain on reduction,

$$
(u-v) \frac{\partial h}{\partial u}+\sum x^{A} \frac{\partial h}{\partial x^{A}}=-h, \quad A=r+1, \cdots, n,
$$

with

$$
v=\sum e_{A}\left(x^{A}\right)^{2} .
$$

The equation (14) has as solution

$$
h=\frac{1}{R} \phi\left(\frac{x^{r+1}}{R}, \cdots, \frac{x^{n}}{R}\right) .
$$

In case $r=n, h=a / R$, with $a$ constant, and the $V_{n}$ is flat.*

The finite equations for the group $\left[V_{\alpha}\right]$ are $\dagger$

$$
x^{\prime i}=\frac{x^{i}-\frac{1}{2} R \delta_{\alpha} e_{\alpha} a_{\alpha}}{1-a_{\alpha} x^{\alpha}+\frac{1}{4} e_{\alpha} e_{\beta} a_{\alpha}^{2}\left(x^{\beta}\right)^{2}} .
$$

4. Subgroups with two types of symbols. We consider in this section the simplest subgroups with two types of symbols. These are:
(e) $\left[P_{\alpha}, S_{\beta \gamma}\right]$,
(f) $\left[P_{\alpha}, U\right]$,
(g) $\left[S_{\alpha \beta}, U\right]$,
(h) $\left[V_{\alpha}, U\right]$,
(i) $\left[S_{\alpha \beta}, V_{\gamma}\right]$.

Each of these we discuss briefly.

(e). The function $h$ has the same form as for (a) since equations (12) are satisfied identically if (9) are.

* L. P. Eisenhart, Riemannian Geometry, p. 85.

$\dagger$ Lie, loc. cit., p. 350. 
(f). Using the form of $h$ for (a) in (11), we see that $h$ is homogeneous of degree -1 in $x^{r+1}, \cdots, x^{n}$, that is, we may write

$$
h=\frac{1}{x^{r+1}} \phi\left(\frac{x^{r+2}}{x^{r+1}}, \ldots, \frac{x^{n}}{x^{r+1}}\right) .
$$

If $r=n$, there is no solution.

(g). If we substitute for $h$ in (11) its value as determined from (c), we obtain

$$
2 u \frac{\partial h}{\partial u}+x^{A} \frac{\partial h}{\partial x^{A}}=-h, \quad A=r+1, \cdots, n .
$$

Hence,

$$
h=\frac{1}{u^{1 / 2}} \phi\left(\frac{x^{r+1}}{u^{1 / 2}}, \ldots, \frac{x^{n}}{u^{1 / 2}}\right) .
$$

(h). Equations (11) and (13) show $\partial h / \partial x^{\alpha}=0$, so that $h$ is the same as in (f). If $r=n$, there is no solution.

(i). For (d), we have seen that (13) imply (11), that is, the form of $h$ for (i) is the same as that for (d).

5. Subgroups with three and four types of symbols, Of the four possibilities $\left[P_{\alpha}, S_{\beta \gamma}, V_{\delta}\right],\left[P_{\alpha}, S_{\beta \gamma}, U\right],\left[P_{\alpha}, V_{\beta}, U\right],\left[S_{\alpha \beta}, V_{\gamma}, U\right]$, only the second and fourth give subgroups:
(j) $\left[P_{\alpha}, S_{\beta \gamma}, U\right]$,
(k) $\left[S_{\alpha \beta}, V_{\gamma}, U\right]$.

For $(\mathbf{j})$, the $P_{\alpha}, S_{\beta \gamma}$ imply $h=h\left(x^{r+1}, \cdots, x^{n}\right)$, and then $U$ shows $h$ is the same form as in (f). There is no solution of $r=n$.

The form of $h$ for (k) will be the same for (h), as follows from (i), that is, $h$ will have the same form as for (f). If $r=n$, there is no solution.

The simplest four type symbol subgroup is

$$
\left[P_{\alpha}, V_{\beta}, S_{\gamma \delta}, U\right] \text {. }
$$

It is easily seen that the solution for $h$ is the same as for (f), and there is no solution for $r=n$.

6. Indices in different ranges. So far, we have considered only subgroups whose symbol indices all have the same range, $1, \cdots, r$. In this section we discuss cases (e), (i), (j), (k), and (l) with the indices for the various types of symbols in different ranges.

Case $(\mathrm{m}):\left[P_{i}, S_{j k}\right]$. Let $i$ range through $1, \cdots, r$, and $j, k$ through any set of $t$ indices, $s_{1}, s_{2}, \cdots, s_{t}$, with $s_{1}<s_{2}<\cdots<s_{t}$. Then either: 
$\left(\mathrm{m}_{1}\right) \quad s_{t} \leqq r, \quad\left(\mathrm{~m}_{2}\right) \quad s_{1} \leqq r, s_{t}>r, \quad\left(\mathrm{~m}_{3}\right) \quad s_{1}>r$.

For case $\left(\mathrm{m}_{1}\right)$, equations (9) imply (12) with $\alpha, \beta$ in the range $s_{1}, s_{2}, \cdots, s_{t}$. Hence $h$ has the same form as in (a).

In the second case, $\left(\mathrm{m}_{2}\right)$, there must be a common index in $(1, \cdots, r)$ and $\left(s_{1}, \cdots, s_{t}\right)$, say $\beta$. Then, in (8c), choose $i=j=\beta$, and $k=s_{t}$. This gives

$$
\left(P_{\beta}, S_{\beta s^{\prime}}\right)=e_{\beta} P_{s^{\prime}}, \quad s^{\prime}=s_{t},
$$

which is not in the set $\left[P_{\alpha}\right]$. Hence, this case is impossible.

For case $\left(m_{3}\right)$, the two sets of indices have no index in common, and we must have $t \geqq 2$. Without loss of generality, we may take the set $s_{1}, \cdots, s_{t}$ to be $r+1, r+2, \cdots, r+t$. The form of $h$ is easily seen to be

$$
h=h\left(v_{t} ; x^{r+t+1}, \cdots, x^{n}\right), \quad v_{t}=\sum_{r+1}^{r+t} e_{J}\left(x^{J}\right)^{2} .
$$

Case $(\mathrm{n}):\left[S_{j k}, V_{i}\right]$. As in case $(\mathrm{m})$, there are three possibilities, only the first and third being possible. If we let $i$ take the range $1, \cdots, r$, then if $s_{t} \leqq r, h$ has the same form as for (d). If $s_{1}>r$, we may let $j, k$ have the range $r+1, \cdots, r+t$. Then $h$ must satisfy (13), and (12) with the indices in this latter range. Since (13) implies (12), we must have $h=h\left(u ; v_{t} ; x^{r+t+1}, \cdots, x^{n}\right)$. Using this form for $h$ in (13), we obtain

$$
(u-w) \frac{\partial h}{\partial u}+v_{t} \frac{\partial h}{\partial v_{t}}+x^{B} \frac{\partial h}{\partial x^{B}}=-h, \quad B=r+t+1, \cdots, n,
$$

with $w=\sum e_{B}\left(x^{B}\right)^{2}$. This equation has as solution

$$
h=\frac{1}{R-v_{t}} \phi\left(\frac{v_{t}}{R-v_{t}} ; \frac{x^{r+t+1}}{R-v_{t}}, \ldots, \frac{x^{n}}{R-v_{t}}\right) .
$$

With three types of symbols, we consider first $\left[P_{i}, S_{j k}, U\right]$, and let $i=1, \cdots, r$. If the indices of $S_{j k}$ are all contained in the range $1, \cdots, r, h$ has the same form as for $\left[P_{\alpha}, U\right]$. Otherwise, we must have all $j, k$ indices outside the range $1, \cdots, r$. Then we have: (o) $\left[P_{a}, S_{J K}, U\right]$, and $h=h\left(v_{t} ; x^{B}\right)$, using the notation of case (n). With this value of $h$ in (11) we obtain equation (15) with $u$ replaced by $v_{t}$. Hence,

$$
h=\frac{1}{v_{t}^{1 / 2}} \phi\left(\frac{x^{r+t+1}}{v_{t}^{1 / 2}}, \cdots, \frac{x^{n}}{v_{t}^{1 / 2}}\right) .
$$

As the next case we consider $\left[V_{\alpha}, S_{j k}, U\right]$. If the $j, k$ indices are included in $1, \cdots, r$, we get the same form for $h$ as in $\left[V_{\alpha}, U\right]$. If not 
we must have $j, k$ in the range $J, K$, to give: (p) $\left[V_{\alpha}, S_{J K}, U\right]$. The symbols $V_{\alpha}, U$ imply $h=h\left(x^{r+1}, \cdots, x^{n}\right)$, and then the symbols $S_{J K}$ imply $h=h\left(v_{t} ; x^{B}\right)$, the same as in (o).

The other two possibilities $\left[P_{i}, S_{j k}, V_{l}\right],\left[P_{i}, V_{j}, U\right]$ are easily shown to be impossible, no matter in what ranges we choose the indices of the various symbols.

For four types we have $\left[P_{\alpha}, S_{j k}, V_{l}, U\right]$. If $j, k$ are in the $J, K$ range, we have a contradiction from $\left(P_{\alpha}, V_{l}\right)$, no matter what range $l$ has. The only other choice is $j, k$ included in the $1, \cdots, r$ range. Then, from $\left(P_{\alpha}, V_{l}\right)$, we must have $l$ in this range also. This gives

(q) $\left[V_{\alpha}, S_{\alpha^{\prime} \beta^{\prime}}, V_{\gamma^{\prime}}, U\right], \quad \alpha^{\prime}, \beta^{\prime}, \gamma^{\prime}$ range included in $1, \cdots, r$, and $h$ has the same form as for (f), as easily follows.

7. Summary. We give here a summary of the various forms for $h$ corresponding to the subgroups considered.
(a) $\left[P_{\alpha}\right]$,
$h=h\left(x^{r+1}, \cdots, x^{n}\right)$;
(b) $[U]$,
$h=\frac{1}{x^{1}} \phi\left(\frac{x^{2}}{x^{1}}, \ldots, \frac{x^{n}}{x^{1}}\right)$;
(c) $\left[S_{\alpha \beta}\right]$,
$h=h\left(u ; x^{r+1}, \cdots, x^{n}\right)$;
(d) $\left[V_{\alpha}\right]$,
$h=\frac{1}{R} \phi\left(\frac{x^{r+1}}{R}, \ldots, \frac{x^{n}}{R}\right)$;
(f) $\left[P_{\alpha}, U\right], \quad h=\frac{1}{x^{r+1}} \phi\left(\frac{x^{r+2}}{x^{r+1}}, \ldots, \frac{x^{n}}{x^{r+1}}\right), r=n$, no solution;
(g) $\left[S_{\alpha \beta}, U\right], \quad h=\frac{1}{u^{1 / 2}} \phi\left(\frac{x^{r+1}}{u^{1 / 2}}, \ldots, \frac{x^{n}}{u^{1 / 2}}\right)$;
$\left(\mathrm{m}_{3}\right)\left[P_{\alpha}, S_{I J}\right], \quad h=h\left(u ; x^{B}\right)$;
(n) $\left[V_{\alpha}, S_{I J}\right], \quad h=\frac{1}{R-v_{t}} \phi\left(\frac{v_{t}}{R-v_{t}} ; \frac{x^{B}}{R-v_{t}}\right)$;
(o $\left.\mathrm{o}_{3}\right)\left[P_{\alpha}, S_{I J}, U\right], h=\frac{1}{v_{t}^{1 / 2}} \phi\left(\frac{x^{B}}{v_{t}{ }^{1 / 2}}\right)$;
(e) $\left[P_{\alpha}, S_{\beta \gamma}\right], \quad$ and $\left(\mathrm{m}_{1}\right) \quad\left[P_{\alpha}, S_{\beta^{\prime} \gamma^{\prime}}\right], \quad h$ as in (a);
(i) $\left[S_{\alpha \beta}, V_{\gamma}\right], \quad$ and $\quad\left(\mathrm{n}_{1}\right) \quad\left[V_{\alpha}, S_{\beta^{\prime} \gamma^{\prime}}\right], \quad h$ as in (d);
(h) $\left[V_{\alpha}, U\right], \quad$ (j) $\left[P_{\alpha}, S_{\beta \gamma}, U\right], \quad$ (k) $\left[S_{\alpha \beta}, V_{\gamma}, U\right]$,
(l) $\left[P_{\alpha}, V_{\beta}, S_{\gamma \delta}, U\right], \quad\left(o_{1}\right) \quad\left[P_{\alpha}, S_{\beta^{\prime} \gamma^{\prime}}, U\right]$,
(p) $\left[V_{\alpha}, S_{\beta^{\prime} \gamma^{\prime}}, U\right], \quad(q) \quad\left[V_{\alpha}, S_{\beta^{\prime} \gamma^{\prime}}, V_{\delta^{\prime}}, U\right]$, 
all have $h$ as in (f);

$$
\left[V_{\alpha}, S_{I J}, U\right], \quad h \text { as in }\left(\mathrm{o}_{3}\right) .
$$

In the above summary we have used the following notation :

$$
R=\sum e_{i}\left(x^{i}\right)^{2}, \quad u=\sum e_{\alpha}\left(x^{\alpha}\right)^{2}, \quad v_{t}=\sum e_{I}\left(x^{I}\right)^{2},
$$

$i=1, \cdots, n$; Greek letters have the range $1, \cdots, r ; I, J=r+1, \cdots$, $r+t ; A=r+1, \cdots, n$; primed Greek letters have a range contained within $1, \cdots, r ; B=r+t+1, \cdots, n$.

8. Restatement of Theorem 2 of I. In the proof of this theorem, the possibility $a_{0}=b^{i}=a_{i}=0$ was omitted. In this case, $\xi^{i}$ has the form $\xi^{i}=b_{j}{ }^{i} x^{j}$, and the function $f(R)$ is arbitrary. The group for this case is evidently the rotation group $\left[S_{i j}\right]$ of $\frac{1}{2} n(n-1)$ parameters. It is not difficult to show that the subgroups corresponding to the two cases mentioned in the theorem are $\left[c e_{i} P_{i}+V_{i}, S_{j k}\right]$ for $f(R)=(\alpha R+\beta)^{2}$ and $\left[S_{i j}, U\right]$ for $f(R)=\alpha R$. We may thus state the corrected theorem in the form:

THEOREM. Every metric space with quadratic form $\sum e_{i}\left(d x^{i}\right)^{2} / f(R)$ admits the rotation group $\left[S_{i j}\right]$ as a group of motions. The only metric spaces with this quadratic form which admit other groups of motions are spaces of constant curvature, and $f$ has the form $f(R)=(\alpha R+\beta)^{2}$, and the group is $\left[c e_{i} P_{i}+V_{i}, S_{j k}\right]$, and spaces with $f(R)=\alpha R$, in which case the group is $\left[S_{i j}, U\right]$.

North Carolina State College 